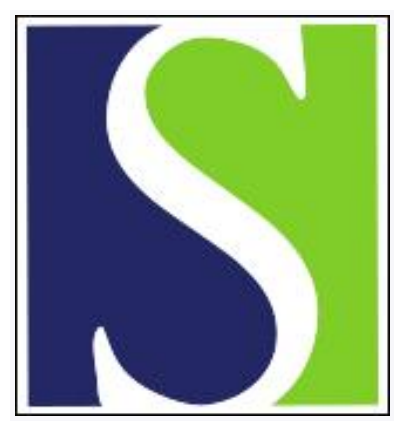

Scand J Work Environ Health 1994;20(4):301-305

https://doi.org/10.5271/sjweh.1394

Issue date: 01 Aug 1994

Parkinsonism after chronic exposure to the fungicide maneb (manganese ethylene-bis-dithiocarbamate).

by Meco G, Bonifati V, Vanacore N, Fabrizio E

Affiliation: Department of Neurosciences, La Sapienza University, Rome, Italy.

This article in PubMed: www.ncbi.nlm.nih.gov/pubmed/7801076 


\title{
Parkinsonism after chronic exposure to the fungicide maneb (manganese ethylene-bis-dithiocarbamate)
}

\author{
by Giuseppe Meco, MD, Vincenzo Bonifati, MD, Nicola Vanacore, BSc, Edito Fabrizio, MD ${ }^{1}$

\begin{abstract}
MECO G, BONIFATI V, VANACORE N, FABRIZIO E. Parkinsonism after chronic exposure to the fungicide maneb (manganese ethylene-bis-dithiocarbamate). Scand J Work Environ Health 1994; $20: 301-5$.

Permanent parkinsonism was observed in a man with chronic exposure to the fungicide maneb (manganese ethylene-bis-dithiocarbamate). Symptoms developed at 37 years of age, two years after exposure had ceased. To our knowledge, this is the second report on parkinsonism associated with exposure to maneb. Manganese is a well-known parkinsonigen toxin in humans. More recently, it has been shown that dithiocarbamates can also induce extrapyramidal syndromes. The biochemical effects of manganese and dithiocarbamates are reviewed and their possible neurotoxic mechanisms are discussed. Both of these components may have played a role in this case.
\end{abstract}

KEY WORDS - dithiocarbamates, manganese.

It has long been known that chronic manganese intoxication induces parkinsonism among miners and some categories of manufacturers exposed to manganese ores $(1-4)$. Symptoms appear after a period of exposure ranging from six months to some years. They include bradykinesia, gait disturbances and postural instability, hypomimia, and postural and (less frequently) rest tremor. Dystonia is often seen in intoxicated miners. Treatments with levodopa (L-dopa) and chelating agents (eg, edetic acid) have sometimes yielded positive results, while they have been ineffective at other times $(1-4)$. The few necropsy studies carried out on manganese-induced parkinsonism have mainly shown degenerative lesions of the globus pallidum and subthalamic nucleus, caudate nucleus, and putamen, with less frequent or less severe lesions of the substantia nigra, a scenario very different from that of idiopathic Parkinson's disease, in which the substantia nigra is typically involved and the strio-pallidal complex is spared (1). Chronic administration of manganese to monkeys also induces an extrapyramidal syndrome with extensive lesions of the basal ganglia (1). At the neurochemical level, most studies have reported a depletion of dopamine in the striatum of animals chronically treated with manganese (1).

More recently several cases of permanent extrapyramidal syndromes have been observed following disulfiram (Antabuse ${ }^{\circledR}$ ) poisoning (5-9). These cases have sometimes been characterized by addi-

1 Department of Neurosciences, "La Sapienza" University, Rome, Italy.

Reprint requests to: Dr G Meco, Department of Neurosciences, Viale dell'Universita' 30, I-00185 Rome, Italy. tional pyramidal signs, peripheral neuropathies, or behavioral abnormalities, and the computed tomography (CT) scans have detected bilateral lesions at the level of the basal ganglia in some of the subjects (7-9).

Ethylene-bis-dithiocarbamate (EBDTC) and carbon disulfide are the main metabolites of disulfiram (10). Carbon disulfide is itself a well-known toxin causing parkinsonism (11), and it can react with endogenous amino acids and monoamines to produce dithiocarbamates (12).

A possible role of $\operatorname{EBDTC}(6,9)$ or carbon disulfide $(7,8)$ has been mentioned in some cases of disulfiram-associated parkinsonism. Moreover, some authors have suggested that EBDTC might be the compound directly responsible for the neurochemical changes and toxicity of disulfiram and carbon disulfide on the extrapyramidal system $(12,13)$.

Besides their role in secondary parkinsonism, it has been hypothesized that manganese (1) and, recently also, dithiocarbamates (6) may also play a role in the pathogenesis of idiopathic Parkinson's disease.

We observed permanent parkinsonism in a man with chronic exposure to the fungicide maneb (manganese ethylene-bis-dithiocarbamate) (figure 1) (14). This compound comprises a wide fungicidal spectrum; it acts mainly through contact with foliage and is used for several crops, including apples, pears, grains, tobacco, and beets. During the last 20 years the consumption of maneb and mancozeb (manganese zinc ethylene-bis-dithiocarbamate) has increased continuously in Italy. In the various marketed preparations containing maneb, the manganese and EBDTC weight ratio is about $1: 4$. Copper is often added to the marketed preparations (14). 


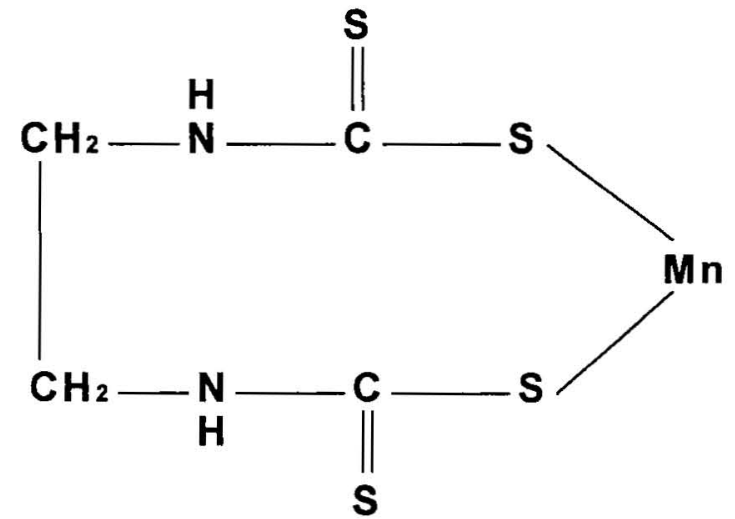

Figure 1. Chemical structure of maneb (manganese ethylene-bis-dithiocarbamate).

\section{Case}

A 47-year-old man came to our center in October 1991. His medical history showed no significant events in the past and no metabolic or neuropsychiatric diseases among relatives. From 23 to 33 years of age he worked in the management and maintenance of the mechanical plant of a malt-producing mill. Then, between 33 and 35 years of age, he planned, constructed, and tested a device for treating barley seeds with fungicides. In particular, during these years, the patient treated about 70 sacks of barley seeds ( $30 \mathrm{~kg}$ each) daily with $5 \mathrm{~g}$ of maneb fungicide in each sack. He was exposed on the average for $4 \mathrm{~h}$ a day, $4 \mathrm{~d}$ a week, 4 months a year. During this time he handled about $45 \mathrm{~kg}$ of fungicides in a closed environment, with a window and ventilation system but without any personal protection (gloves or mask). In 1981, at the age of 37 years, two years after the exposure to maneb had ceased, a mild tremor associated with paresthesias appeared in his right leg and later spread to the ipsilateral arm. A neurologist diagnosed extrapyramidal syndrome and prescribed amantadine $(100 \mathrm{mg}$ ) and trihexyphenidyl $(2 \mathrm{mg})$, which had a beneficial effect on the symptoms. However, during the following three years the tremor worsened, spreading to the contralateral limbs. The symptoms then remained stable for about seven years. Our first neurological examination showed mild, generalized bradykinesia and rigidity, postural tremor in the right limbs, mild tremor of the lips, mild slowness of gait with reduced swinging of the arms, seborrhea, mild hypomimia, and slurred speech. No other neurological signs appeared in the examination.

The blood analyses, the electrocardiogram, and the visual evoked potentials were normal. Cerebrospinal fluid samples were not available due to the patient's refusal to undergo lumbar puncture. The serum copper and ceruloplasmin levels were normal (copper: $105 \mu \mathrm{g} \cdot \mathrm{dl}^{-1}$, normal range $65-165$; ceruloplasmin: $35.1 \mathrm{mg} \cdot \mathrm{dl}^{-1}$, normal range $15-60$ ), Wilson's dis- ease thus being excluded as a cause of the parkinsonism. The urinary activity of arylsulfatase was also in the normal range, a finding excluding another rare metabolic cause of secondary parkinsonism.

The CT scan of the skull was normal, the only exception being a mild enlargement of the right lateral ventricle. The acute administration of apomorphine $(1 \mathrm{mg})$ subcutaneously or L-dopa $(200 \mathrm{mg}$ ) orally had no effects on the symptoms. Thus the chronic treatment with amantadine and trihexyphenidyl was maintained.

In July 1992 the patient returned for a check-up because of a worsening of the symptoms during the last six months. Our examination confirmed the worsening of his symptoms and revealed the presence of rest tremor in all four limbs and the lips, particularly in the upper limbs and on the right side. A magnetic resonance imaging (MRI) scan of the brain was performed with a Magnetom ${ }^{\circledast}$ imager (Siemens) with a 1.5-T magnetic field. The asymmetry of the ventricular system, already seen with the CT, with a mild enlargement of the right lateral ventricle, was confirmed. In proton density and $\mathrm{T}_{2}$-weighted scans some small hyperintense areas appeared in the bilateral frontal and left parietal white matter. These appearances can be interpreted as nonspecific gliotic foci. The basal ganglia were normal (figures $2-3$ ). Finally, a neuropsychological evaluation showed no relevant abnormalities. However, some perseverative errors and some difficulties on the attentive tests and in shifting between semantic and alphabetic categories were noted.

In May 1993, during the last check-up, a further slight worsening of the extrapyramidal symptoms was found. The L-dopa challenge ( $250 \mathrm{mg}$ orally) was repeated, but again it had no effect on the symptoms.

\section{Discussion}

Manganese affects biological systems in numerous ways, but its primary neurotoxic mechanism is still far from clear (15). The metal impairs the functioning of some receptors and ionic channels of the plasma membrane, the systems for signal transduction and second messenger synthesis, some cellular enzymes, and other metalloproteins (15). One particular target of the metal is the mitochondria, where the manganese alters the calcium homeostasis and provokes an oxidative stress. Other mechanisms that have been suggested to explain the neurotoxicity of manganese include dopamine auto-oxidation, stimulation of free radicals and 6-OH-dopamine production, and the reduction of levels of reduced glutathione, glutathione peroxidase and catalase (15).

The extrapyramidal syndromes associated with the disulfiram-EBDTC-carbon disulfide group of compounds are much less studied and much less known. Dithiocarbamates are chelating compounds which form lipophilic complexes with various metallic ions 


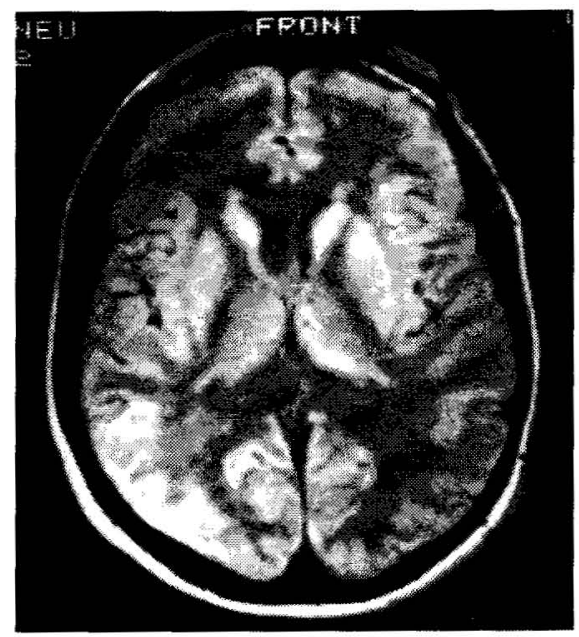

Figure 2. Proton density weighted scan made with magnetic resonance imaging and showing normal basal ganglia.

(13). Such complexes tend to accumulate in the central nervous system (CNS) and in other tissues with high lipid concentrations. EBDTC is one of the most studied dithiocarbamates. It has been proposed as a chelating agent for the treatment of cadmium and nickel intoxication and even for Wilson's disease (13, 16). However it has been shown that the effect of this compound, like that of other chelating agents, is not a simple depletion but rather a redistribution of metals in the body, with a reduction of levels in some organs and an increase in others, including the CNS $(13,16)$.

In particular, the administration of EBDTC to rats, with or without the addition of copper to the diet, increases the levels of copper in the CNS (13). Once they have passed the blood-brain barrier, the EBDTC-metal complexes may dissociate, and the metal may accumulate in the brain. Toxicity then ensues, probably through oxidative reactions and free radical production (13). This mechanism probably explains why the pretreatment of rats with EBDTC enhances the toxic effects of 1-methyl-4-phenyl1,2,3,6-tetrahydropyridine (MPTP), a selective toxin for the dopaminergic pathways, responsible for cases of permanent human parkinsonism $(17,18)$. Moreover, EBDTC inhibits some enzymes which need metal ions to function, for example, superoxide dismutase, a scavenger enzyme for superoxide radicals (19) and dopamine- $\beta$-hydroxylase (12).

As regards maneb, only one case of acute intoxication by a mixture of maneb and zineb (zinc ethylene-bis-dithiocarbamate) has been reported, with transient loss of consciousness, convulsions, and hemiparesis, all of which disappeared after a few days (20).

In 1988 two cases of parkinsonism were first observed in young agricultural workers exposed to various fungicides, though mainly to maneb, for

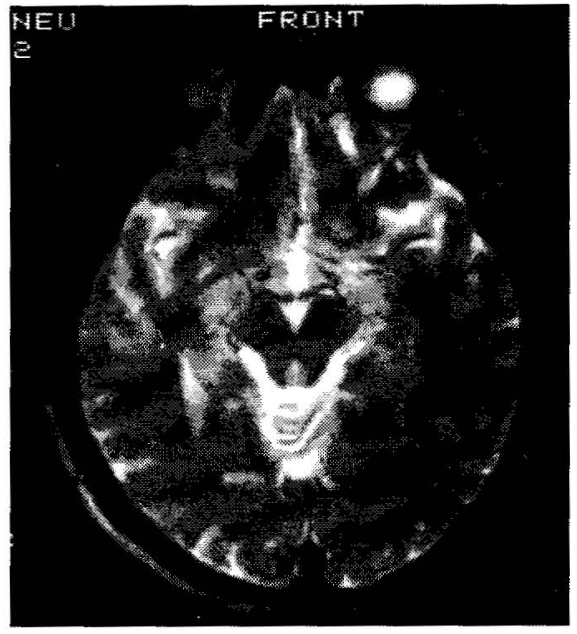

Figure 3. $T_{2}$-weighted scan made with magnetic resonance imaging and showing normal mesencephalic structures.

four to five years (21). After this observation, a group of 69 workers from the same region was screened. The results showed a significantly increased frequency of rigidity with cogwheel phenomenon, headache, fatigue, nervousness, memory complaints, and sleepiness in the people exposed to maneb. Postural tremor, bradykinesia, and cerebellar signs were also seen in the exposed group but without statistical significance. The authors stated that occupational exposure to pesticides containing manganese may induce signs of CNS manganese intoxication, and thus the role played by EBDTC in fungicide toxicity may be less important than that of manganese (21).

This study represents the second report of parkinsonism associated with chronic exposure to maneb. The early onset (age 37 years) and the stabilization of symptoms after an initial worsening suggest that some environmental factors might have played a role in this case. Moreover, the intense, chronic and "selective" exposure to the fungicide maneb makes this case very interesting.

In light of the biochemical effects of manganese and EBDTC, a toxic cooperative mechanism can be hypothesized that is probably based on the accumulation of heavy metals in the CNS (13).

The latency of two years observed in this case between the end of exposure to the fungicide and the onset of extrapyramidal symptoms must be stressed. To our knowledge, in manganese intoxications, such a phenomenon has never been reported. Moreover, after the exposure to manganese has ceased, the symptoms may improve, but they usually become stable and occasionally continue to worsen $(1-4)$. There is only one reported case in which mild gait disturbances appeared during exposure to manganese with the main extrapyramidal symptoms developing three years after the exposure had ceased (2). 
For our case we hypothesized that the accumulation, induced by maneb, of heavy metals like manganese and copper in the CNS could have primed a form of delayed toxicity responsible for the delayed onset of symptoms. Indeed, in light of the effects of EBDTC on the brain, it has been stated that EBDTC and disulfiram treatments could enhance the risk of neurological diseases many years later (13). Moreover, delayed effects have been claimed to explain other cases of dithiocarbamate toxicity (6). As an alternative hypothesis, for this patient, the chronic exposure to maneb might have interacted with and accelerated a preexisting degenerative process, leading to the early clinical appearance of parkinsonism.

For our patient a determination of blood or hair manganese levels was not performed. It is known that a relationship between manganese body levels and neurological symptoms does not exist $(1,21)$. Besides, such a determination, as well as that for EBDTC, would be of little relevance years after the exposure had ceased.

The lack of effects of L-dopa or apomorphine and the beneficial effects of amantadine and trihexyphenidyl on this patient suggest the presence of lesions distal to the nigrostriatal pathway, in accordance with the results of a positron emission tomographic study on manganese-induced parkinsonism (22).

The CT and MRI scans showed no relevant findings in our case. Bilateral pallidoputaminal lesions have been detected by CT (7-9) and MRI (7) in some cases of extrapyramidal syndromes after severe disulfiram intoxication. As regards manganese-induced parkinsonism, the MRI was abnormal in only one of the four cases examined (22).

Last, the neuropsychological examination of our patient detected, in the context of good global performance, some perseverative errors and attentive difficulties, findings compatible with an initial involvement of the frontal lobe functions and in accordance with the cognitive pattern of parkinsonian patients (23).

Besides the involvement of manganese in forms of secondary parkinsonism, which are different from idiopathic Parkinson's disease on a pathological lev$\mathrm{el}$, the role of manganese, as well as that of dithiocarbamates in the etiopathogenesis of Parkinson's disease, is still unclear. Barbeau (1) proposed that initial damage to the nigrostriatal system, caused by a variety of factors (including manganese intoxication) but able to induce a critical increase in dopamine turnover, could trigger a neurotoxic selfperpetuating mechanism leading, through the production of oxidative stress in the surviving cells, to the progressive loss of the dopaminergic neurons and to the development of Parkinson's disease.

After the discovery of MPTP-induced parkinsonism (17), numerous studies have been devoted to the search for environmental toxins in Parkinson's disease etiopathogenesis (24). Some epidemiologic sur- veys have identified risk factors such as living in rural areas, the use of well water, and exposure to pesticides for Parkinson's disease (24). Another casereferent study found instead that working in orchards and planer mills, two activities involving exposure to fungicides, increased the risk of Parkinson's disease (25).

In conclusion, this is the second report of permanent parkinsonism observed after chronic exposure to the fungicide maneb. Both of its components (manganese and EBDTC) are potential toxins for the extrapyramidal system and might have had toxic effects. A better comprehension of the way these substances act on basal ganglia may help to clarify the mechanisms of delayed neurotoxicity and contribute to the understanding of the pathogenesis of other extrapyramidal disorders like Parkinson's disease, dystonias, and Wilson's disease.

\section{Acknowledgments}

Part of this study was supported by a grant from MURST (Italian Ministry for Scientific and Technological Research) (60\%).

We thank Dr L Baker for his linguistic assistance.

\section{References}

1. Barbeau A. Manganese and extrapyramidal disorders. Neurotoxicology 1984;5:13-36.

2. Cook DG, Fahn S, Brait KA. Chronic manganese intoxication. Arch Neurol 1974;30:59-64.

3. Huang CC, Chu NS, Lu CS, Wang JD, Tsai JL, Tzeng JL, et al. Chronic manganese intoxication. Arch Neurol 1989;46:1104-6.

4. Mena I, Marin O, Fuenzalida S, Cotzias GC. Chronic manganese poisoning - clinical picture and manganese turnover. Neurology 1967;17:128-36.

5. Fontan M, Petit H, Delahousse J, Ascher J, DecoulxPoutignat M. Signes extrapyramidaux d'intoxications aigues ou subaigues par disulfiram ou disulfiram-alcool. Lille Med 1967;12:814-7.

6. Hoogenraad TU. Dithiocarbamates and Parkinson's disease [letter]. Lancet 1988;1:767.

7. Krauss JK, Mohadjer M, Wakhloo AK, Mundinger F. Dystonia and akinesia due to pallidoputaminal lesions after disulfiram intoxication. Movement Disord 1991; 6:166-70.

8. Laplane D, Attal N, Sauron B, de Billy A, Dubois B. Lesions of basal ganglia due to disulfiram neurotoxicity. J Neurol Neurosurg Psychiatry 1992;55:9259.

9. Lidy C, Priollet P, Pepin B. Syndrome pyramidal et extra-pyramidal apparu au décours d'une intoxication aiguë par le disulfiram [letter]. Nouv Presse Med 1979;8:3561.

10. Rainey JM. Disulfiram toxicity and carbon disulfide poisoning. Am J Psychiatry 1977;134:371-8.

11. Peters HA, Levine RL, Matthews CG, Chapman LJ. Extrapyramidal and other neurologic manifestations associated with carbon disulfide fumigant exposure. Arch Neurol 1988;45:537 40.

12. McKenna MJ, DiStefano V. Carbon disulfide: II. a proposed mechanism for the action of carbon disulfide on dopamine $\beta$-hydroxylase. J Pharmacol Exp Ther 1977;202:253-66.

13. Allain P, Krari N. Diethyldithiocarbamate, copper, and neurological disorders. Life Sci 1991;48:291-9. 
14. Fishbein L. Environmental health aspects of fungicides: I. dithiocarbamates. J Toxicol Environ Health 1976;1:713-35.

15. Aschner M, Aschner JL. Manganese neurotoxicity: cellular effects and blood-brain barrier transport. Neurosci Biobehav Rev 1991;15:333-40.

16. Weiss B, Cory-Slechta DA, Cox C. Modification of lead distribution by diethyldithiocarbamate. Fundam Appl Toxicol 1990;15:791-9.

17. Ballard PA, Tetrud JW, Langston JW. Permanent human parkinsonism due to 1-methyl-4-phenyl-1,2,3,6tetrahydropyridine (MPTP): seven cases. Neurology 1985;35:949-56.

18. Corsini GU, Pintus S, Chiueh CC, Weiss JF, Kopin IJ. 1-Methyl-4-phenyl-1,2,3,6-tetrahydropyridine (MPTP) neurotoxicity in mice is enhanced by pretreatment with diethyldithiocarbamate. Eur J Pharmacol 1985;119:127-8.

19. Heikkila RE, Cabbat FS, Cohen G. In vivo inhibition of superoxide dismutase in mice by diethyldithiocarbamate. J Biol Chem 1976;251:2182-5.

20. Israeli $R$, Sculsky $M$, Tiberin P. Acute central nervous system changes due to intoxication by Manzidan (a combined dithiocarbamate of maneb and zineb). Arch Toxicol 1983; suppl 6:238-43.

21. Ferraz HB, Bertolucci PHF, Pereira JS, Lima JGC, Andrade LAF. Chronic exposure to the fungicide maneb may produce symptoms and signs of CNS manganese intoxication. Neurology 1988;38:550-3.

22. Wolters EC, Huang CC, Clark C, Peppard RF, Okada $\mathrm{J}$, Chu NS, et al. Positron emission tomography in manganese intoxication. Ann Neurol 1989;26:647 51 .

23. Gotham AM, Brown RG, Marsden CD. "Frontal" cognitive function in patients with Parkinson's disease "on" and "off" levodopa. Brain 1988;111:299-321.

24. Tanner CM. The role of environmental toxins in the etiology of Parkinson's disease. Trends Neurosci 1989;12:49-54.

25. Hertzman C, Wiens M, Bowering D, Snow B, Calne D. Parkinson's disease: a case-control study of occupational and environmental risk factors. Am J Ind Med 1990;17:349-55.

Received for publication: 26 July 1993 